\title{
Survival prediction models for patients with anal carcinoma receiving definitive chemoradiation: A population-based study
}

\author{
YINHANG WU ${ }^{1}$, XIAOYANG HAN ${ }^{2}$, YAN LI $^{3}$, KUNLI ZHU $^{1 *}$ and JINMING YU ${ }^{1 *}$ \\ ${ }^{1}$ Department of Radiation Oncology, Shandong Cancer Hospital Affiliated to Shandong University, Jinan, Shandong 250000; \\ ${ }^{2}$ Department of Oncology, Shandong Provincial Hospital Affiliated to Shandong University, Jinan, Shandong 250021; \\ ${ }^{3}$ Clinical Laboratory, Huaiyin District Center for Disease Control and Prevention, Jinan, Shandong 250022, P.R. China
}

Received May 31, 2019; Accepted November 8, 2019

DOI: $10.3892 / 01.2019 .11238$

\begin{abstract}
The present study aimed to develop two nomograms in order to predict cancer-specific survival (CSS) and overall survival (OS) of patients with anal carcinoma receiving definitive chemoradiotherapy. Data from studies including patients with anal carcinoma, who were determined to be positive histologically and diagnosed between 2004 and 2010, were obtained from the Surveillance, Epidemiology, and End Results database. Significant prognostic factors for CSS and OS of patients were screened to develop nomograms through univariate and multivariate analyses. Nomograms were validated using internal and external data. The predictive abilities of the generated models were evaluated by concordance index (C-index) and calibration curves. Risk stratification was performed for patients with the same TNM stage. A total of 1,473 patients and six independent prognostic factors for CSS and OS, namely age, sex, ethnicity, marital status at diagnosis, $\mathrm{T}$ stage and $\mathrm{N}$ stage, were included in the nomogram calculations. Calibration curves demonstrated that nomogram prediction was in high accordance with actual observation. The $\mathrm{C}$-indices of nomograms were greater than those of models based on the sixth edition of the American Joint Committee on Cancer TNM staging system for CSS prediction (training cohort, 0.72 vs. 0.70 ; validation cohort, 0.68 vs. 0.62 ) and $O S$ (training cohort, 0.70 vs. 0.66 ; validation cohort, 0.68 vs. 0.62 ). Survival curves demonstrated significant survival differences among the different risk groups. Nomograms were more accurate than the conventional TNM staging system in prognosis
\end{abstract}

Correspondence to: Professor Jinming Yu or Dr Kunli Zhu, Department of Radiation Oncology, Shandong Cancer Hospital Affiliated to Shandong University, 440 Jiyan Road, Jinan, Shandong 250000, P.R. China

E-mail: sdyujinming@163.com

E-mail: 2664123676@qq.com

${ }^{*}$ Contributed equally

Key words: anal carcinoma, chemoradiation, nomogram, risk stratification, Surveillance, Epidemiology, and End Results, survival prediction. In addition, survival performances of patients with the same TNM stage could be further distinguished by risk stratification, which provided individualized prediction for patients. These survival prediction methods may aid clinicians in patient counseling and in selecting more individualized therapeutic strategies.

\section{Introduction}

Anal carcinoma accounts for $1.9 \%$ of digestive system cancer cases, according to a report from $2009(1,2)$. Currently, the standard therapeutic method for locally advanced anal carcinoma is concurrent chemoradiotherapy (3-6). Although the TNM classification system is widely recognized and used as a reliable method for the identification of the clinical stage of a variety of cancer types and consequent improvement of diagnostic accuracy and therapeutic effect, the survival outcome of patients with the same type of cancer and TNM stage is not always similar in certain types of cancer, including non-small and small cell lung cancer (7-9). It has been considered that the TNM classification system may not be the sole decisive factor for the survival status of patients with cancer (7-9). Other factors, including age, sex, histology and treatment strategies, may also affect the survival outcome of patients with cancer (7-9).

Nomogram development has been regarded as a convenient and reliable method to predict individualized probability of survival in patients with cancer by scoring risk factors (10-16). The predictive accuracy of nomograms has been found to be higher than that of the TNM classification system in some types of cancer, including non-small cell lung cancer, nasopharyngeal cancer and non-metastatic head and neck squamous cell carcinoma $(13,17,18)$. However, the application of nomograms in patients with anal carcinoma receiving definitive chemoradiotherapy has not yet become available. Cancer-specific survival (CSS) is the length of time from the date of cancer diagnosis to the date of death from the disease. Overall survival (OS) corresponds to the duration between patients' diagnosis with cancer and the death from any causes. The present study aimed to develop two reliable models and nomograms to predict the CSS and OS of patients with anal carcinoma, who underwent definitive chemoradiotherapy. Furthermore, the Cox proportional hazard regression model 
was externally validated by assessing data from a separate cohort of patients with anal cancer diagnosed between 2011 and 2012 in the Surveillance, Epidemiology, and End Results (SEER) database.

\section{Materials and methods}

Data retrieval and processing. The SEER program (https://seer. cancer.gov/) is a reliable and recognized database maintained by the National Cancer Institute that consists of diagnostic and therapeutic data collected from patients with various types of cancer. The original data of patients with anal carcinoma diagnosed histologically between 2004 and 2010 were released in November 2017 and extracted from the SEER database using the SEER*Stat software (version 8.3.5) (https://seer.cancer. gov/seerstat/). In total, 10,073 records were searched using this software. Any patients with histologically confirmed anal carcinoma and tumor sites confined to the anus, anal canal or anorectum, diagnosed between 2004 and 2010, were considered. The inclusion criteria were as follows: i) Only one primary malignant tumor; ii) T1-T4, N0-N3, M0 confirmed by MRI according to the sixth edition of the American Joint Committee on Cancer TNM classification (19); iii) chemoradiotherapy without surgery and no history of cancer-directed surgery of primary site, lymph nodes or other relative sites; iv) known ethnicity, histology type, marital status at diagnosis and cause of death; and v) active follow-up with complete data and known survival outcome. Additionally, only the following pathological types were included: i) Squamous cell carcinoma; ii) basaloid carcinoma; and iii) cloacogenic carcinoma. Other types of carcinoma, including adenocarcinoma and neuroendocrine carcinoma, were excluded due to small sample sizes or different therapeutic strategies. As a continuous variable, age was transformed into a categorical variable according to recognized cut-off values to minimize the loss of information (20). In addition, a separate cohort, which met the aforementioned inclusion criteria, was retrieved using the SEER*Stat program from the SEER database based on eligible patients that were diagnosed between 2011 and 2012, and considered as the external validation cohort in order to assess the generalizability of the models.

Nomogram development. Univariate analysis for different variables, including age, sex, ethnicity, marital status at diagnosis, $\mathrm{T}$ category, $\mathrm{N}$ category, stage category, histology and grade, was conducted in the training cohort using the Kaplan-Meier estimates. Furthermore, survival curves were plotted and log-rank tests were performed to ensure the reliability of the results. Variables with $\mathrm{P}<0.05$, which was considered to indicate a statistically significant difference, were entered into the multivariate analysis using the Cox proportional hazard regression model to exclude confounding factors. The univariate and multivariate analyses were conducted using SPSS software (version 20.0; IBM Corp.). Variance inflation factor was calculated with the R statistical package (version 3.5.2; R Core Team; http://www.r-project.org) prior to constructing the model to prevent overfitting caused by multicollinearity. The nomograms, which were based on the eligible results of the multivariate Cox proportional hazard regression model, were constructed using the 'rms' package (version 5.1-3) for $\mathrm{R}$ software (21). The Cox proportional hazard regression model was selected according to a backward step-down process based on the Akaike Information Criterion (22).

Validation and calibration of the nomograms compared with the TNM classification system. The performance of the final model was assessed by calculating the concordance index (C-index) and plotting the calibration curves of 1-, 3- and 5 -year CSS and OS after performing the internal validation via 1,000 bootstrap resamples and external validation using the validation cohort, respectively (23). The C-index values ranged between 0.5 and 1.0, which was in accordance with the predictive ability of the models. The C-indices of the different models were compared using the 'survcomp' package (version 1.32.0) $(24,25)$.

Risk stratification and prediction of survival probability. Discrimination ability was compared between the nomogram and standard TNM staging system based on the C-indices and the unique ability of nomogram was evaluated by risk stratification. The patients were divided into different risk groups in accordance with cut-off values of the total risk score that were determined using X-tile software (version 3.6.1) (26). These cut-off values were subsequently used for the external validation cohort and Kaplan-Meier survival curves of the corresponding risk groups were plotted. The survival probability of each patient was predicted.

\section{Results}

Basic data characteristics. Based on the aforementioned search conditions, 10,073 records were retrieved from the SEER database. In order to be included in the present study, patients had to meet all inclusion criteria. In total, 1,473 and 638 eligible patients were included in the training and external validation cohorts, respectively. The clinicopathological characteristics and demographics of the eligible patients in both cohorts are presented in Table SI.

Univariate and multivariate analyses of the training cohort. Univariate and multivariate analyses for CSS and OS were conducted in the training cohort to select significant and independent risk factors. The results of the analysis and eligible variables included in the final Cox proportional hazard regression model are shown in Tables SII and SIII. The variance inflation factor results are shown in Table SIV and indicated the normal range as $0-10$ (Table SIV).

Nomogram development for CSS and OS. Nomograms were constructed based on the incorporation of significant and independent prognostic factors, including age, sex, ethnicity, marital status at diagnosis, $\mathrm{T}$ stage and $\mathrm{N}$ stage. Two nomograms are displayed in Fig. 1. The contributions of relative covariates to nomogram prediction of the CSS of patients with anal carcinoma were ranked in descending order: i) $\mathrm{T}$ category; ii) $\mathrm{N}$ category; iii) age; iv) sex; v) ethnicity; and vi) marital status at diagnosis. Furthermore, the contributions of relative covariates to nomogram prediction of the OS of patients were ranked in descending order: i) $\mathrm{T}$ category; ii) age; iii) $\mathrm{N}$ category; iv) sex; v) ethnicity; and vi) marital 
A

Points

Age

Sex

Ethnicity

Marital.status

T6th

N6th

Total Points

1-year CSS

3-year CSS

5-year CSS

B

Points

Age

Sex

Ethnicity

Marital.status

T6th

N6th

Total Points

1-year OS

3-year OS

5-year OS
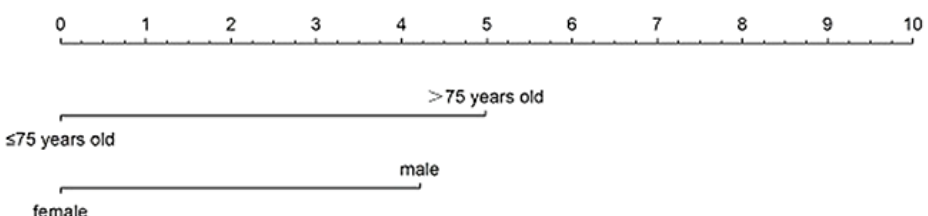

female
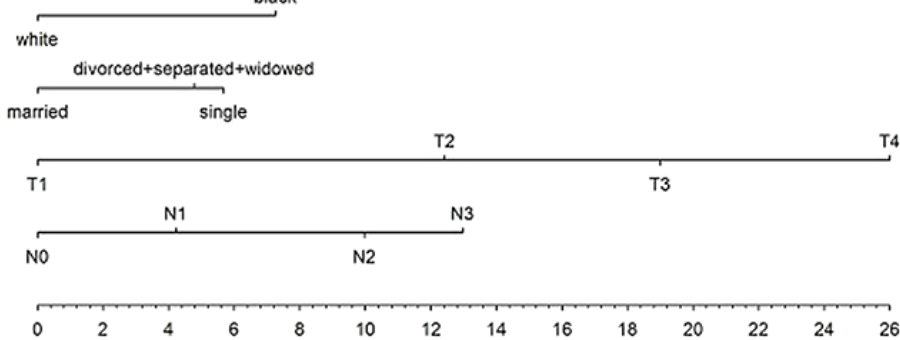

\begin{tabular}{lllll}
\hline 0.95 & 0.85 & 0.75 & 0.65
\end{tabular}

\begin{tabular}{lllllllll}
\hline 0.95 & 0.85 & 0.75 & 0.65 & 0.55 & 0.45 & 0.35 & 0.25 & 0.15
\end{tabular}

$\begin{array}{lllllllll}0.95 & 0.85 & 0.75 & 0.65 & 0.55 & 0.45 & 0.35 & 0.25 & 0.15\end{array}$

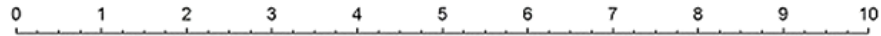

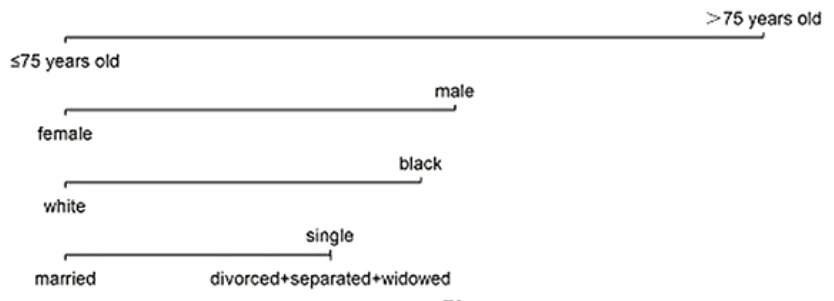

divorced+separated
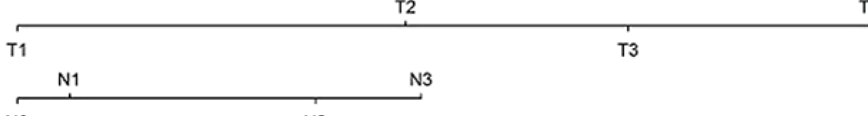

NO N2

\begin{tabular}{lllllll}
\hline 0 & 5 & 10 & 15 & 20 & 25 & 30
\end{tabular}

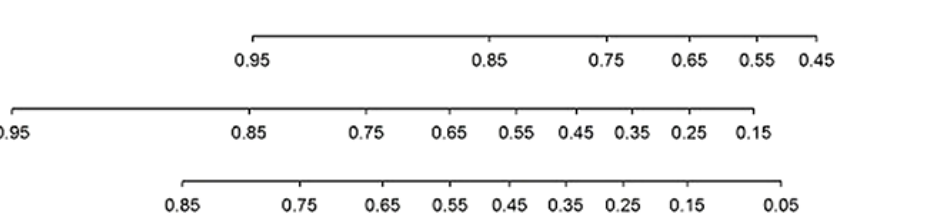

Figure 1. Nomogram for the prediction of 1-, 3- and 5-year CSS and OS. (A) Nomogram A, including age, sex, ethnicity, marital status, T6th and N6th, for 1-, 3- and 5-year CSS in patients with anal carcinoma. (B) Nomogram B, including age, sex, ethnicity, marital status, T6th and N6th, for 1-, 3- and 5-year OS in patients with anal carcinoma. The nomograms provide the probability of 1-, 3- and 5-year CSS and OS in combination with covariates. For instance, if 'Age' is located and a vertical line drawn upwards to the 'Points' axis, the corresponding score belongs to 'Age'. Other variables can be found by repeating the same process. Subsequently, the total score of each patient can be calculated by summing up the score of each variable. According to the total score of each patient, the location can be identified at the 'Total Points' axis and a vertical line can be drawn downwards in order to find the probability of 1-, 3- and 5-year CSS or OS. T6th, T stage according to the sixth edition of the AJCC tumor-node-metastasis staging system; N6th, N stage according to the sixth edition of the AJCC tumor-node-metastasis staging system; CSS, cancer-specific survival; OS, overall survival; AJCC, American Joint Committee on Cancer.

status at diagnosis. Additionally, the scores of these variables in different nomograms were unequal. The total score of each patient was calculated by summing up the score of each subtype within these variables in the nomogram to predict the probability of individualized survival.

Calibration and validation of the nomograms. In the training cohort, the C-index of the nomogram for CSS was $0.72(95 \%$ CI, 0.69-0.75; $\mathrm{P}<0.05)$ and that of the nomogram for OS was 0.70 (95\% CI, 0.68-0.72; $\mathrm{P}<0.05)$, respectively. In addition, the
C-index of the TNM classification system for CSS was 0.70 (95\% CI, 0.67-0.73; P<0.05) and that of the TNM classification system for OS was 0.66 (95\% CI, 0.63-0.69; $\mathrm{P}<0.05)$. In the external validation cohort, the $\mathrm{C}$-index of the nomogram for CSS was $0.68(95 \% \mathrm{CI}, 0.62-0.73 ; \mathrm{P}<0.05)$ and that of the nomogram for OS was 0.68 (95\% CI, 0.63-0.73; $\mathrm{P}<0.05)$, respectively. Furthermore, the $\mathrm{C}$-index of the TNM classification system for CSS was 0.62 (95\% CI, 0.56-0.69; $\mathrm{P}<0.05)$ and that of the TNM classification system for OS was 0.62 (95\% CI, 0.56-0.67; P<0.05). However, the C-index of the 
A

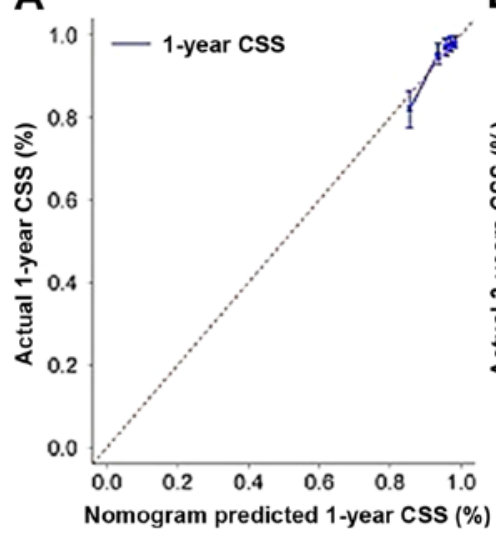

B

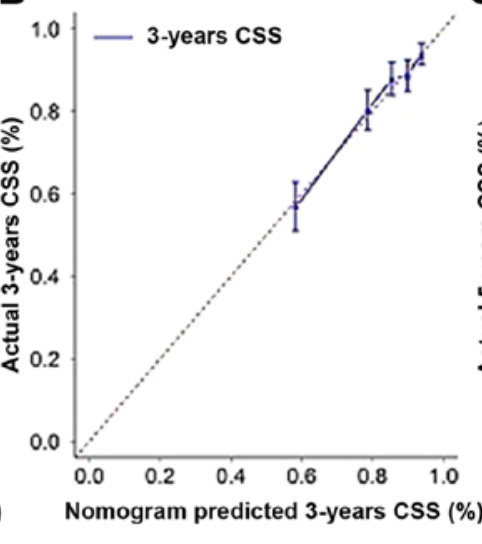

C

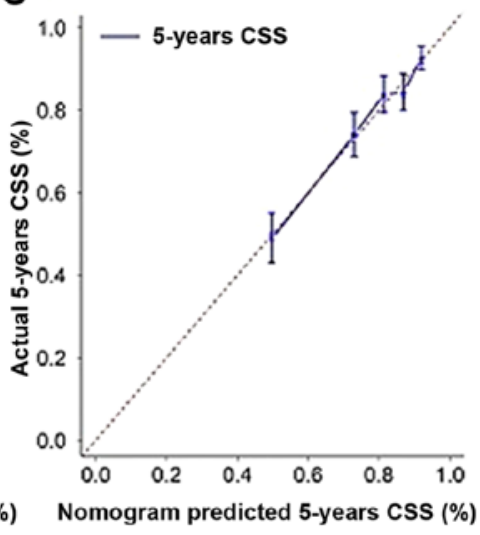

D

E
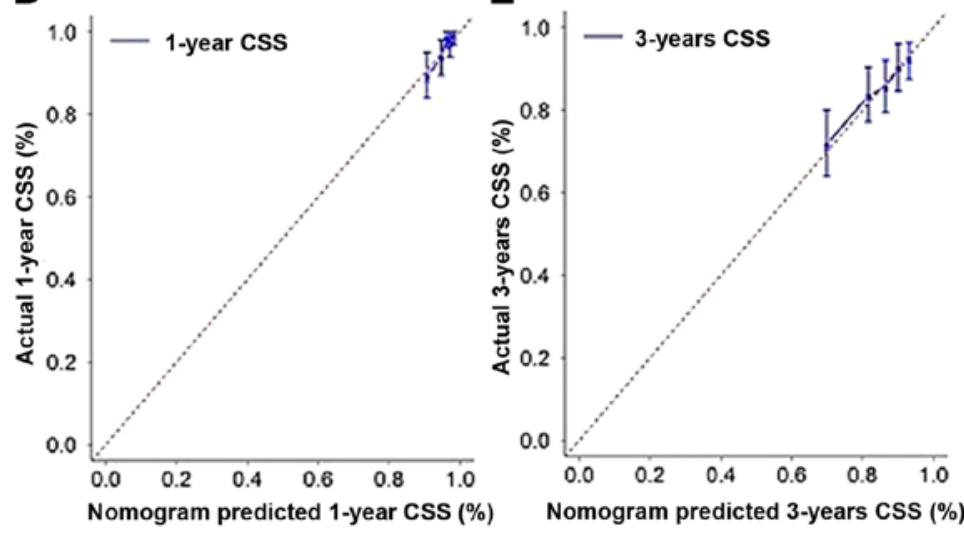

$\mathbf{F}$

G

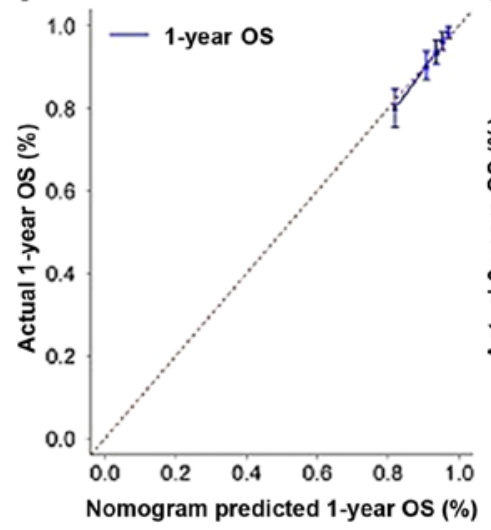

$1.0+$ - 3-years os

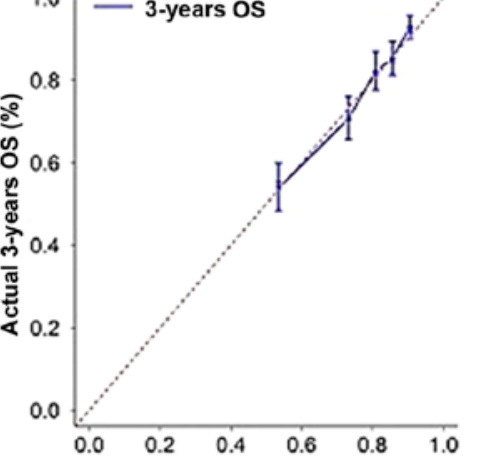

H

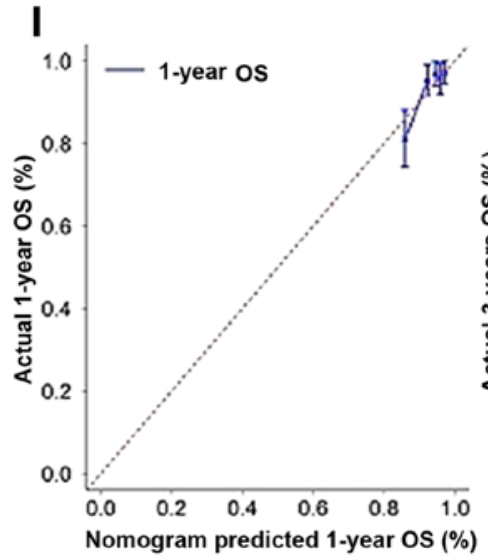

$\mathrm{J}$

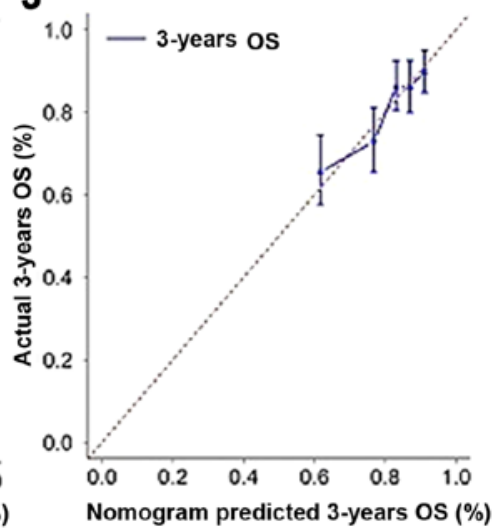

Figure 2. Bootstrap calibration of nomograms in the training cohort and external validation cohort Calibration curves of nomogram A for predicting CSS at (A) 1, (B) 3 and (C) 5 years in the training cohort, and at (D) 1 and (E) 3 years in the external validation cohort. The calibration curves of nomogram B for predicting OS at (F) $1,(\mathrm{G}) 3$ and $(\mathrm{H}) 5$ years in the training cohort, and at (I) 1 and $(\mathrm{J}) 3$ years in the external validation cohort. Nomogram-predicted CSS or OS was plotted on the x-axis; actual CSS or OS was plotted on the y-axis. A plot along the 45-degree line demonstrates optimal agreement between nomogram-predicted survival and actual survival. CSS, cancer-specific survival; OS, overall survival. 
A
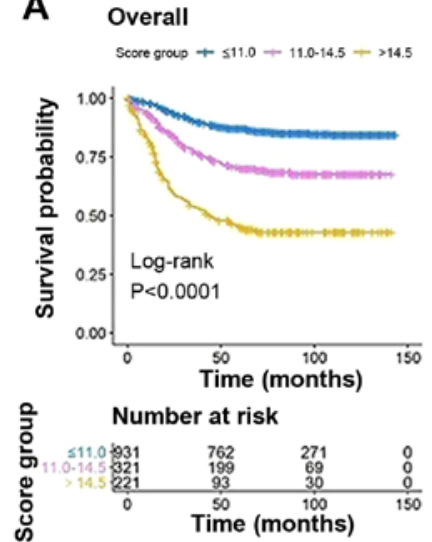

D Stage IIIA

Score group + s11.0 $+11.0-14.5+>14.5$
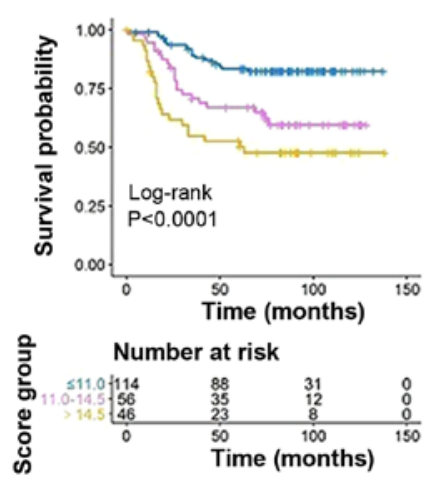

F Overall
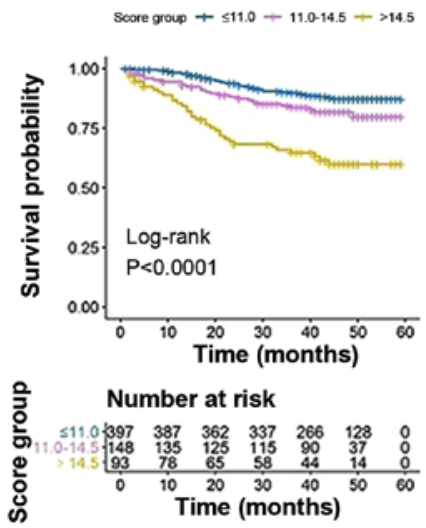

I Stage IIIA

Score group $+\leq 11.0+11.0 .14 .5+>14.5$
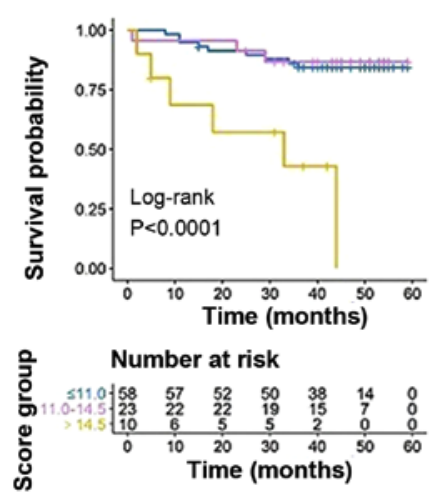

B Stage I

Score group $+\$ 11.0+11.0 \cdot 14.5$
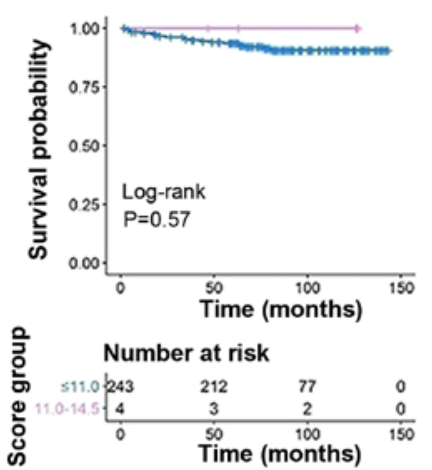

E Stage IIIB

Score group + s11.0 $+11.0-14.5+>14.5$
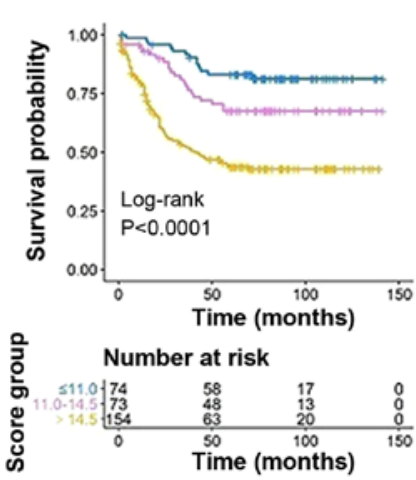

G Stage I
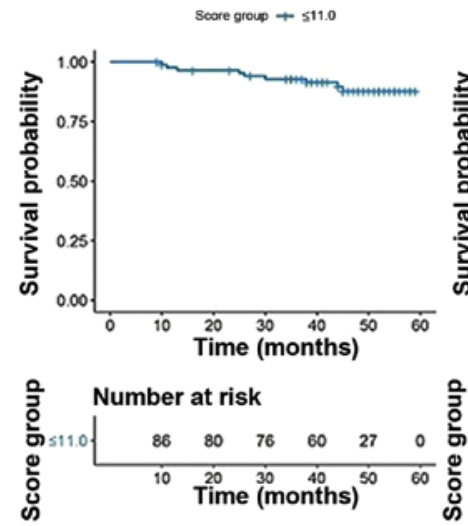

J Stage IIIB

score group $+\$ 11.0+11.0-14.5+>14.5$
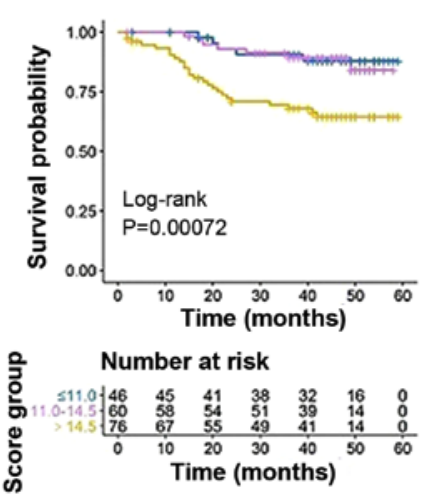

Figure 3. Risk group stratification for cancer-specific survival. (A-E) Risk group stratification based on nomogram A within corresponding TNM stage for (A) total patients and (B-E) stages I-IIIB in the training cohort. (F-J) Risk group stratification based on nomogram A within corresponding TNM stage for (F) total patients and (G-J) stages I-IIIB in the external validation cohort. 
A

Overall
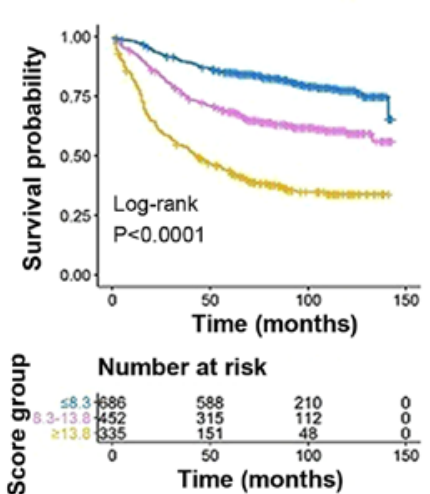

D Stage IIIA

score group $+\leq 8.3+8.3 \cdot 13.8+213.8$
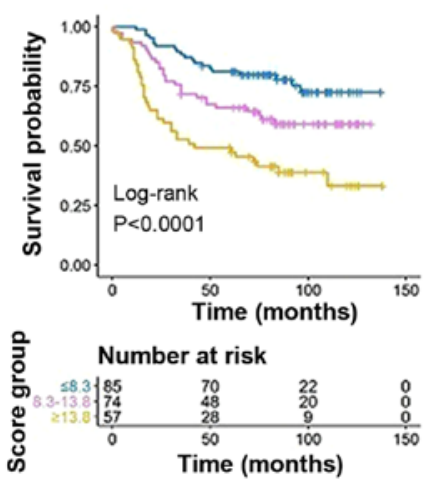

F Overall

Scove group $+\leq 8.3+8.3 \cdot 13.8+213.8$
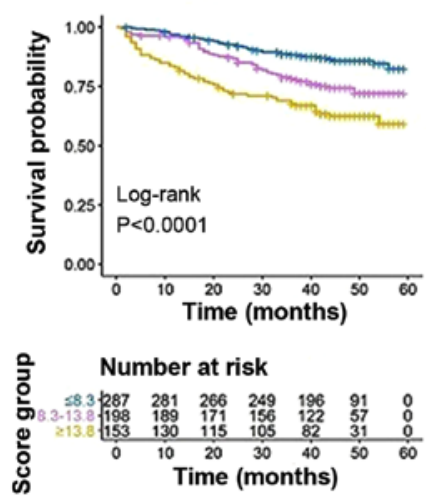

I Stage IIIA

Score group $+\leq 8.3+8.3 \cdot 13.8+213.8$
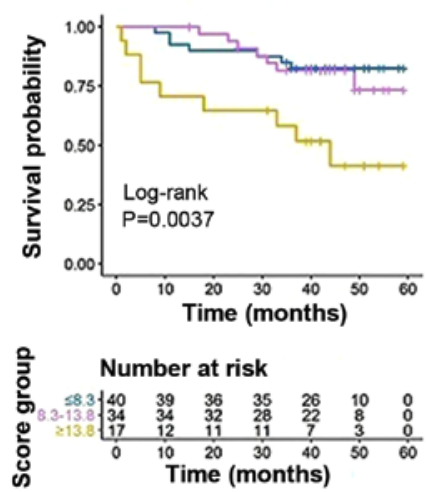

B Stage I
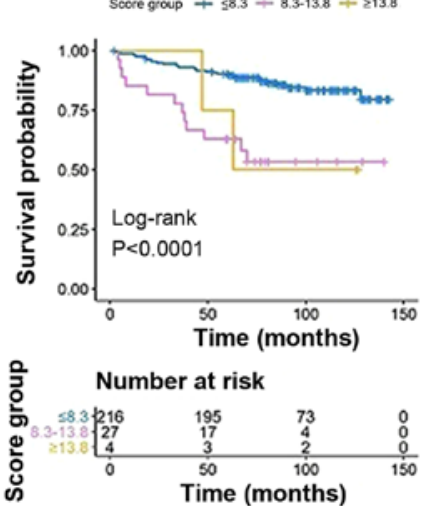

E Stage IIIB

Scoce group $+58.3+8.3 \cdot 13.8+213.8$
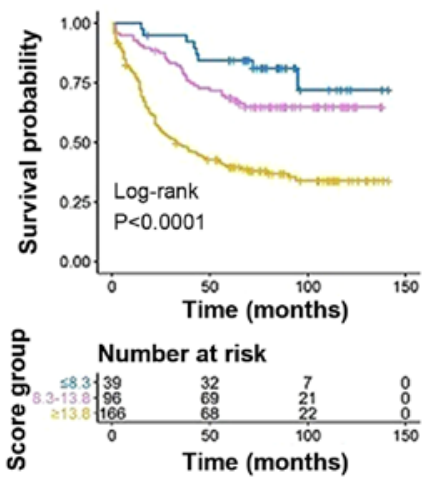

G Stage I
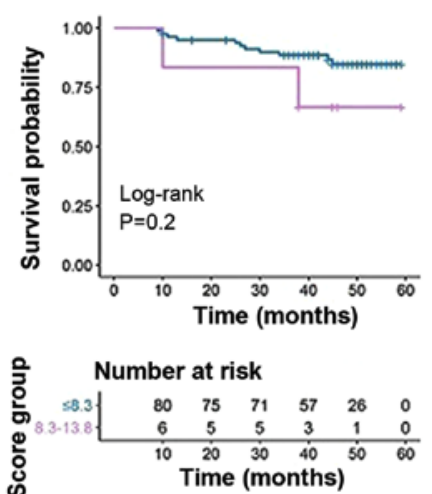

J Stage IIIB
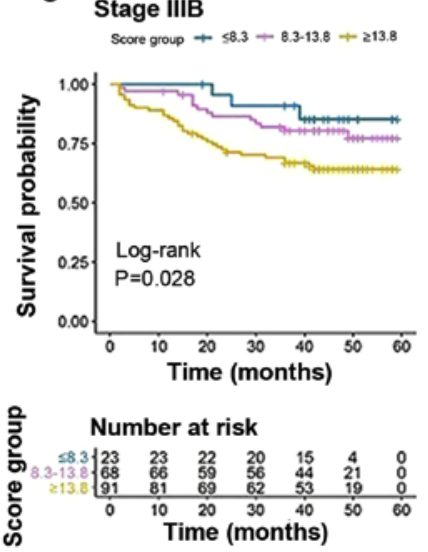

C Stage II
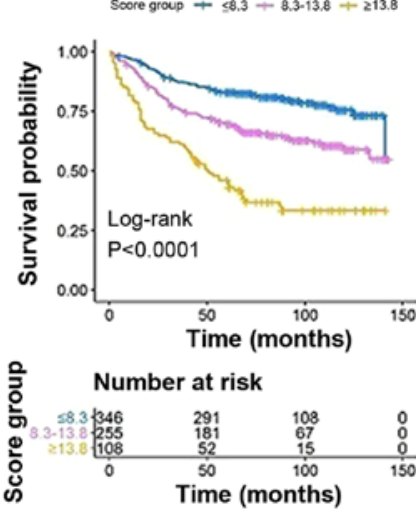

Time (months)
H Stage II

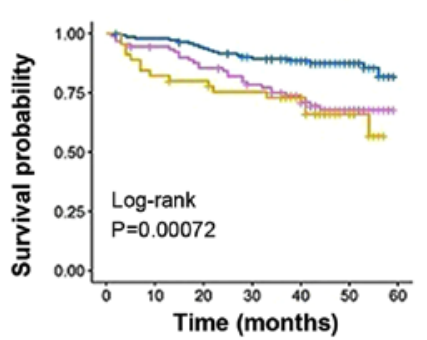

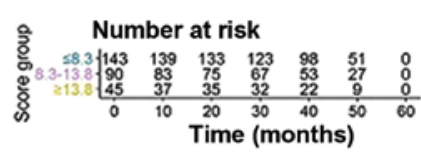

Time (months) 
nomogram predicting CSS was higher than that of the TNM staging system predicting CSS (training cohort, 0.72 vs. 0.70 , respectively; $\mathrm{P}=0.07$; external validation cohort, 0.68 vs. 0.62 , respectively; $\mathrm{P}<0.05$ ). Similarly, the $\mathrm{C}$-index of the nomogram predicting OS was also significantly higher than that of the TNM staging system predicting OS (training cohort, 0.70 vs. 0.66 , respectively; $\mathrm{P}<0.05$; external validation cohort, 0.68 vs. 0.62, respectively; $\mathrm{P}<0.05$ ). Results from the calibration curves revealed excellent agreement between nomogram prediction and actual observation for 1-, 3- and 5-year CSS and OS in the training cohort (Fig. 2). Additionally, this agreement was similarly demonstrated in the calibration curves of the external validation cohort (Fig. 2).

Performance of risk stratification and prediction of survival probability. Patients in the training cohort were divided into three risk groups according to the cut-off values of the total risk scores derived from the corresponding nomogram. The prognostic conditions of patients from each risk group are presented in Table SV and Figs. 3 and 4. Following the application of these cut-off values to the external validation cohort, the survival differences of patients stratified into different risk groups were clearly demonstrated by the Kaplan-Meier curves within each stage category (Figs. 3 and 4). The 1-, 3- and 5 -year survival probability of each patient was predicted and presented in Tables SVI and SVII.

\section{Discussion}

Anal carcinoma accounts for $1.9 \%$ of digestive system cancer cases, according to the 2009 report $(1,2)$ and concurrent chemoradiotherapy is the current standard therapeutic method for locally advanced anal carcinoma (3-6). The application of nomograms has been reported in survival time prediction of patients with several types of cancer, including cervical cancer, prostate cancer, rectal cancer, non-small-cell lung cancer and breast cancer (10-14). However, to the best of our knowledge, the use of nomograms as reliable tools for the prediction of CSS and OS of patients with anal carcinoma receiving definitive chemoradiotherapy has not yet been reported. According to previous studies, the survival time of patients with different types of cancer is markedly varied and the survival outcome of patients with the same type of carcinoma and TNM stage also varies widely, indicating that the TNM classification system is limited with regards to predicting the individualized survival of patients with cancer (7-9). An accurate prognostic system capable of reflecting the prognosis of these patients is required. Therefore, nomograms based on a large number of patient data obtained from the SEER database were generated to predict individualized survival. Risk stratification was performed to further distinguish prognosis for patients with the same TNM stage in the present study.

The training and external validation cohorts were formulated based on diagnostic and therapeutic data of patients with a variety of cancer types retrieved from the SEER database and currently maintained by the National Cancer Institute. A total of 2,111 eligible patients, specifically 1,473 in the training cohort and 638 in the external validation cohort, were analyzed. Age, sex, ethnicity, marital status at diagnosis, $\mathrm{T}$ category and $\mathrm{N}$ category were selected and considered as significant prognostic factors, independent to the CSS and OS of patients with anal carcinoma, through univariate and subsequent multivariate analyses. The results of univariate analysis regarding the stage variable were statistically significant; however, this variable was excluded due to evident association with the $\mathrm{T}$ category prior to multivariate analysis. These findings were in accordance with previous studies concerning risk factors for anal carcinoma, which in indicated that patients with anal carcinoma have a higher risk of mortality if they are male, of higher age and black $(27,28)$. Apart from common risk factors in certain types of cancer, including prostate cancer and oropharyngeal carcinoma $(29,30)$, including age or sex, marital status at diagnosis was also identified as an independent prognostic factor in patients with anal carcinoma in the present study and affected the CSS and OS. A previous study also indicated that unmarried patients were at significantly higher risk of presenting with metastatic cancer, undertreatment and cancer-associated mortality (31). Though chemoradiation is considered the standard treatment for epidermoid anal cancer due to the findings of the first UK Co-ordinating Committee on Cancer Research Anal Cancer Trial (1996) and subsequent 13-year follow-up study $(6,32)$, the outcome of chemoradiation used as a treatment for anal adenocarcinoma is not equal to anal squamous cell carcinoma (33). Therefore, adenocarcinoma was excluded from the present study. According to previous studies $(34,35)$, certain rare pathological types of anal cancer, including basaloid carcinoma and cloacogenic carcinoma, were also taken into consideration. Nomograms were constructed based on the results of multivariate analysis. To prevent overfitting of the model and to improve generalizability, the validation of the model is indispensable (36). According to the C-indices of the model and the calibration curves for CSS and OS, the prediction results of the established nomograms closely corresponded to the actual observation. The $\mathrm{C}$-indices of nomograms for CSS and OS in the training cohort were $>0.7$, which suggested good predictive precision regarding the survival time of patients. The calibration curves revealed optimal agreement between nomogram prediction and actual observation in the training and external validation cohorts, which indicated the reliability of the established model. Additionally, although calibration curves were only plotted for the probability of 1- and 3-year CSS and OS for the external validation cohort, which was due to the lack of 5-year survival data after 2012 in the SEER database, the performance of these calibration curves was satisfactory and demonstrated a good degree of fit between nomogram prediction and actual outcome. Compared with the conventional TNM staging system, the $\mathrm{C}$-indices of nomograms were significantly higher in the CSS and OS of both cohorts, which suggested the superiority of nomograms. It was concluded that the prognosis of patients with the same TNM stage varied widely as shown by the significantly different survival performances of risk groups within the respective TNM stages. The difference of stage IIIA and IIIB between low- and moderate-risk groups in the training cohort (Fig. 3D and E) was more evident than that of the external validation cohort (Fig. 3I and J). This result was mainly affected by the small sample size of patients with stage IIIA and IIIB anal carcinoma in the external validation cohort. 
Although the classical TNM staging system is widely used in the clinical setting, the survival outcome of patients with cancer is not only influenced by the tumor itself. Additional factors, including age, sex and therapeutic strategies, may affect the prognosis of patients with certain types of cancer, including urothelial carcinoma (37). The development of nomograms, which are practical and convenient tools that evaluate the survival probability of a specific outcome, and include large influential factors and easily available and measurable parameters, could successfully overcome the disadvantages of the conventional TNM staging system $(38,39)$.

Nomogram development may contribute to individualized management decisions in the use of concurrent chemoradiotherapy for patients with anal carcinoma, and risk stratification may lead to improvement of the current treatment strategies. For example, a 77 year-old, black, male patient with anal carcinoma (T3NOM0) was found to have 24.10 points and a $17 \% 5$-year probability of OS in the generated nomogram. Furthermore, a 40 year-old, white, female patient with anal carcinoma (T3NOM0) was found to have 7.17 points and a $80 \% 5$-year probability of OS in the constructed nomogram. The therapeutic strategies and clinical decisions made for these patients were not identical due to the significant survival difference. By contrast, both patients would be diagnosed with stage II anal carcinoma according to the conventional TNM staging system, which would predict identical prognosis.

However, the present study had certain limitations. First, the sample size used for constructing the nomograms was relatively small due to the low morbidity of anal carcinoma and strict inclusion criteria. Secondly, the retrospective information from the SEER database may have contributed to the risk of potential selection bias in the established nomograms. Thirdly, the present study did not include data from Asian populations due to limitations of the SEER database. Finally, not all potential prognostic factors associated with survival were included in the nomograms. For example, performance status, nutritional conditions, comorbidities and financial issues were excluded factors because they were not available. A larger sample size and more comprehensive information, including performance status, nutritional status, comorbidity factors and financial issues, would be required to further improve the predictive ability of the generated survival model in future studies.

In conclusion, two nomograms were constructed to predict the individualized survival of patients with anal carcinoma receiving definitive chemoradiotherapy for the first time. The nomograms were demonstrated to have higher accuracy than the conventional TNM staging system in prognosis prediction and the survival performances of patients with the same TNM stage could be further distinguished by risk stratification. Therefore, nomograms were capable of providing individualized prediction for patients with anal carcinoma, and may aid clinical decision-making and the selection of more individualized therapeutic strategies according to the specific needs of each patient.

\section{Acknowledgements}

Not applicable.

\section{Funding}

The present study was supported by the Key Research and Development Project of Shandong Province (grant no. 2017GSF18158).

\section{Availability of data and materials}

The datasets generated and/or analyzed during the current study are available in the SEER database (https://seer.cancer.gov/).

\section{Authors' contributions}

YW extracted data and analyzed most of the data. XH contributed to data analysis. YL performed data interpretation. KZ contributed to the funding support, participated in the design of the study and wrote the manuscript. JY designed the study. All authors approved the final version of the manuscript.

\section{Ethics approval and consent to participate}

Not applicable.

\section{Patient consent for publication}

Not applicable.

\section{Competing interests}

The authors declare that they have no competing interests.

\section{References}

1. Jemal A, Siegel R, Ward E, Hao Y, Xu J and Thun MJ: Cancer statistics, 2009. CA Cancer J Clin 59: 225-249, 2009.

2. Karanjawala BE and Chang GJ: Management of Anal Cancer, 2015.

3. Nieder C: Concurrent radiochemotherapy with 5-FU/mitomycin remains standard treatment for anal carcinoma. Long-term results of the phase III RTOG 98-11 trial. Strahlenther Onkol 189: 512-513, 2013 .

4. Nigro ND: An evaluation of combined therapy for squamous cell cancer of the anal canal. Dis Colon Rectum 27: 763-766, 1984.

5. Franco P, Mistrangelo M, Arcadipane F, Munoz F, Sciacero P, Spadi R, Migliaccio F, Angelini V, Bombaci S, Rondi N, et al: Intensity-modulated radiation therapy with simultaneous integrated boost combined with concurrent chemotherapy for the treatment of anal cancer patients: 4-year results of a consecutive case series. Cancer Invest 33: 259-266, 2015.

6. Epidermoid anal cancer: Results from the UKCCCR randomised trial of radiotherapy alone versus radiotherapy, 5-fluorouracil, and mitomycin. UKCCCR Anal Cancer Trial Working Party. UK Co-ordinating Committee on Cancer Research. Lancet 348: 1049-1054, 1996.

7. Giroux D, Meerbeeck JV, Crowley JJ, Sculier JP, Chansky K and Goldstraw PJCJoLC: The international association for the study of lung cancer staging project, 13: 792-801, 2010.

8. Kawaguchi T, Takada M, Kubo A, Matsumura A, Fukai S, Tamura A, Saito R, Maruyama Y, Kawahara M and Ignatius Ou SH: Performance status and smoking status are independent favorable prognostic factors for survival in non-small cell lung cancer: A comprehensive analysis of 26,957 patients with NSCLC. J Thorac Oncol 5: 620-630, 2010.

9. Sculier JP, Chansky K, Crowley JJ, Van Meerbeeck J and Goldstraw P; International Staging Committee and Participating Institutions: The impact of additional prognostic factors on survival and their relationship with the anatomical extent of disease expressed by the 6th Edition of the TNM Classification of Malignant Tumors and the proposals for the 7th Edition. J Thorac Oncol 3: 457-466, 2008. 
10. Kim MK, Jo H, Kong HJ, Kim HC, Kim JW, Kim YM, Song YS, Kang SB, Mok JE and Lee HP: Postoperative nomogram predicting risk of recurrence after radical hysterectomy for early-stage cervical cancer. Int J Gynecol Cancer 20: 1581-1586, 2010.

11. Stephenson AJ, Scardino PT, Eastham JA, Bianco FJ Jr, Dotan ZA, DiBlasio CJ, Reuther A, Klein EA and Kattan MW: Postoperative nomogram predicting the 10 -year probability of prostate cancer recurrence after radical prostatectomy. J Clin Oncol 23: 7005-7012, 2005

12. van Stiphout RG, Valentini V, Buijsen J,Lammering G, Meldolesi E, van Soest J, Leccisotti L, Giordano A, Gambacorta MA, Dekker A and Lambin P: Nomogram predicting response after chemoradiotherapy in rectal cancer using sequential PETCT imaging: A multicentric prospective study with external validation. Radiother Oncol 113: 215-222, 2014.

13. Liang W, Zhang L, Jiang G, Wang Q, Liu L, Liu D, Wang Z, Zhu Z, Deng Q, Xiong X, et al: Development and validation of a nomogram for predicting survival in patients with resected non-small-cell lung cancer. J Clin Oncol 33: 861-869, 2015.

14. Van Zee KJ, Manasseh DM, Bevilacqua JL, Boolbol SK, Fey JV, Tan LK, Borgen PI, Cody HS III and Kattan MW: A Nomogram for predicting the likelihood of additional nodal metastases in breast cancer patients with a positive sentinel node biopsy. Ann Surg Oncol 10: 1140-1151, 2003

15. Wang Y, Li J, Xia Y, Gong R, Wang K, Yan Z, Wan X, Liu G, Wu D, Shi L, et al: Prognostic nomogram for intrahepatic cholangiocarcinoma after partial hepatectomy. J Clin Oncol 31: 1188-1195, 2013.

16. Egelmeer AG, Velazquez ER, de Jong JM, Oberije C, Geussens Y, Nuyts S, Kremer B, Rietveld D, Leemans CR, de Jong MC, et al: Development and validation of a nomogram for prediction of survival and local control in laryngeal carcinoma patients treated with radiotherapy alone: A cohort study based on 994 patients. Radiother Oncol 100: 108-115, 2011

17. Jian JP, Ng WT, Jing FZ, Lee SWM, Choi HCW, Chan LL, Lin SJ, Guo QJ, Sze HC, Chen YB, et al: Prognostic nomogram for refining the prognostication of the proposed 8th edition of the AJCC/UICC staging system for nasopharyngeal cancer in the era of intensity-modulated radiotherapy. Cancer 122: 3307-3315, 2016.

18. Xu C, Chen YP, Liu X, Tang LL, Chen L, Mao YP, Zhang Y, Guo R, Zhou GQ, Li WF, et al: Socioeconomic factors and survival in patients with non-metastatic head and neck squamous cell carcinoma. Cancer Sci 108: 1253-1262, 2017.

19. Greene FL, Page DL, Fleming ID, et al: AJCC Cancer Staging Manual: TNM Classification of Malignant Tumors. 6th ed. New York, NY, Springer-Verlag, 2002.

20. Lothar K and Hermsen O: Median split, k-group split, and optimality in continuous populations. AStA Adv Stat Analysis 94: 53-74, 2010

21. Frank E and Harrell Jr: RMS: Regression modeling strategies. R package version 3: 4-0. http://www.rproject.org/

22. Harrell FE Jr, Lee KL and Mark DB: Multivariable prognostic models: Issues in developing models, evaluating assumptions and adequacy, and measuring and reducing errors. Stat Med. Stat Med 15: 361-387, 1996.

23. Rao SJJPotASA: Regression modeling strategies: With applications to linear models, logistic regression, and survival analysis. (Book Reviews) 98: 257-258, 2005.

24. Kang L, Chen W, Petrick NA and Gallas BD: Comparing two correlated $\mathrm{C}$ indices with right-censored survival outcome: $\mathrm{A}$ one-shot nonparametric approach. Stat Med 34: 685-703, 2015.

25. Hanley JA and McNeil BJ: A method of comparing the areas under receiver operating characteristic curves derived from the same cases. Radiology 148: 839-843, 1983.
26. Camp RL, Dolled-Filhart M and Rimm DL: X-Tile A New Bio-informatics tool for biomarker assessment and outcome-based Cut-point optimization. Clin Cancer Res 10: 7252-7259, 2004.

27. Bilimoria KY, Bentrem DJ, Rock CE, Stewart AK, Ko CY and Halverson A: Outcomes and prognostic factors for squamous-cell carcinoma of the anal canal: Analysis of patients from the national cancer data base. Dis Colon Rectum 52: 624-631, 2009.

28. Tsikitis VL, Lu KC, Kim JS, Billingsley KG, Thomas CR Jr and Herzig DO: Nomogram for predicting overall survival and salvage abdominoperineal resection for patients with anal cancer. Dis Colon Rectum 59: 1-7, 2016.

29. Rigaud J, Le Normand L, Karam G, Glemain P, Buzelin JM and Bouchot O: Prognostic factors of prostate cancer treated with first-line hormone therapy. Prog Urol 12: 232-239, 2002 (In French).

30. Rios Velazquez E, Hoebers F, Aerts HJ, Rietbergen MM, Brakenhoff RH, Leemans RC, Speel EJ, Straetmans J, Kremer B and Lambin P: Externally validated HPV-based prognostic nomogram for oropharyngeal carcinoma patients yields more accurate predictions than TNM staging. Radiother Oncol 113: 324-330, 2014.

31. Aizer AA, Chen MH, McCarthy EP, Mendu ML, Koo S, Wilhite TJ, Graham PL, Choueiri TK, Hoffman KE, Martin NE, et al: Marital status and survival in patients with cancer. J Clin Oncol 31: 3869-3876, 2013.

32. Northover J, Glynne-Jones R, Sebag-Montefiore D, James R, Meadows H, Wan S, Jitlal M and Ledermann J: Chemoradiation for the treatment of epidermoid anal cancer: 13-year follow-up of the first randomised UKCCCR Anal Cancer Trial (ACT I). Br J Cancer 102: 1123-1128, 2010.

33. Papagikos M, Crane CH, Skibber J, Janjan NA, Feig B, Rodriguez-Bigas MA, Hung A, Wolff RA, Delclos M, Lin E and Cleary K: Chemoradiation for adenocarcinoma of the anus. Int J Radiat Oncol Biol Phys 55: 669-678, 2003.

34. Bertani E, Chiappa A, Mazzarol G, Contino G, Lazzari R, Zampino MG, Viale G and Andreoni B: Aggressive treatment approach for cloacogenic carcinoma of the anorectum: Report from a single cancer center. Dig Surg 27: 297-301, 2010.

35. Nakagawa R, Eguchi R, Ota T, Yoshitoshi K, Noguchi T and Suzuki T: A case of basaloid cell carcinoma of the anal canal in which complete response was obtained by chemoradiation therapy. Nihon Shokakibyo Gakkai Zasshi 110: 419-425, 2013 (In Japanese).

36. Iasonos A, Schrag D, Raj GV and Panageas KS: How To build and interpret a nomogram for cancer prognosis. J Clin Oncol 26: 1364-1370, 2008.

37. Pond GR, Agarwal N, Bellmunt J, Choueiri TK, Qu A, Fougeray R, Vaughn D, James ND, Salhi Y, Albers P, et al: A nomogram including baseline prognostic factors to estimate the activity of second-line therapy for advanced urothelial carcinoma. BJU Int 113: E137-E143, 2014.

38. Kattan MW, Stapleton AM, Wheeler TM and Scardino PT: Evaluation of a nomogram used to predict the pathologic stage of clinically localized prostate carcinoma. Cancer 79: 528-537, 1997.

39. Kutikova A, Smaldone MC, Egleston BL, Manley BJ, Canter DJ, Simhan J, Boorjian SA, Viterbo R, Chen DY, Greenberg RE and Uzzo RG: Anatomic features of enhancing renal masses predict malignant and high-grade pathology: A preoperative nomogram using the RENAL nephrometry score. Eur Urol 60: 241-248, 2011.

This work is licensed under a Creative Commons Attribution-NonCommercial-NoDerivatives 4.0 International (CC BY-NC-ND 4.0) License. 